\title{
Aqueous Interleukin-6 (IL-6) Level Is a Marker for Treatment Resistance to Bevacizumab in Age-Related Macular Degeneration
}

\author{
-Aqueous Cytokines after Bevacizumab
}

\author{
Kakarla V. Chalam, Sandeep Grover, Sankarathi Balaiya, Ravi K. Murthy ${ }^{*}$ \\ Department of Ophthalmology, University of Florida College of Medicine, Jacksonville, USA. \\ Email: ravi.keshavamurthy@jax.ufl.edu
}

Received November $16^{\text {th }}, 2013$; revised January $12^{\text {th }}, 2014$; accepted February $8^{\text {th }}, 2014$

Copyright (c) 2014 Kakarla V. Chalam et al. This is an open access article distributed under the Creative Commons Attribution License, which permits unrestricted use, distribution, and reproduction in any medium, provided the original work is properly cited. In accordance of the Creative Commons Attribution License all Copyrights (C) 2014 are reserved for SCIRP and the owner of the intellectual property Kakarla V. Chalam et al. All Copyright (C) 2014 are guarded by law and by SCIRP as a guardian.

\section{ABSTRACT}

Background: To prospectively evaluate the effect of intravitreal bevacizumab on aqueous levels of interleukin-6 (IL-6) and vascular endothelial growth factor (VEGF) in patients with exudative age-related macular degeneration (AMD) and to correlate clinical outcomes of patients and aqueous cytokine levels before and after injection. Methods: The study group consisted of 30 eyes from 30 patients with exudative AMD who underwent intravitreal injection of bevacizumab three times at monthly intervals. The aqueous samples prior to the 1st injection (baseline) and 3rd injection were analyzed for VEGF and IL-6 levels, evaluating the effect of 2 doses of intravitreal bevacizumab. Study patients were sub-grouped based upon change in central subfield (CSF) macular thickness on SD-OCT, at 8 weeks. Group 1 included patients $(n=14)$ with a decrease in CSF thickness greater than $10 \%$ from the baseline and were categorized to have "improved". Group 2 included patients $(n=16)$ who had a decrease in CSF thickness $\mathbf{1 0} \%$ or less and were considered "treatment-resistant". Results: There was no statistically significant change in aqueous VEGF and IL-6 levels after intravitreal bevacizumab. In sub-group analysis, in both Groups 1 and 2 patients, aqueous IL-6 levels showed a better correlation with CSF thickness on SD-OCT ( $r=0.72$ and 0.71 , respectively). Conclusions: Data from our study suggest that aqueous IL-6 may be an important marker of treatment response or resistance. Future therapeutic strategies may include targeted treatment against both VEGF and IL-6, in patients who do not respond to anti-VEGF treatment alone.

\section{KEYWORDS}

VEGF; Interleukin-6; AMD; Bevacizumab; Aqueous; Bead Based Assay

\section{Introduction}

Age-related macular degeneration (AMD) is the leading cause of irreversible blindness among patients over the age of 55 years in the western world [1,2]. Exudative $\mathrm{AMD}$, a less common form, is characterized by the formation of a choroidal neovascular membrane that emanates from the choriocapillaris through a defective Bruch's membrane [3]. Vascular endothelial growth factor (VEGF), a diffusible cytokine that induces endotheli-

"Corresponding author. al cell proliferation and leakage has been implicated as an important factor in the neovascular process and associated exudation, hemorrhage, and subsequent development of detachment of the neurosensory retina and retinal pigment epithelium (RPE) [4,5].

Inhibition of VEGF has become a widely accepted treatment modality of exudative AMD [6,7]. Bevacizumab, an anti-VEGF agent, binds and inhibits VEGF related cellular effects [8]. In addition to VEGF, choroidal neovascularization (CNV) involves a number of angiogenic molecules and inflammatory cytokines: IL-6 (In- 
terleukin-6), IL-8 (Interleukin-8), ICAM-1 (Intercellular adhesion molecule-1) and MCP-1 (monocyte chemoattractant protein-1) $[9,10]$.

IL-6, a multifunctional cytokine, is implicated in angiogenesis along with VEGF in a variety of in-vitro and cancer studies [11,12]. High levels of IL-6 in blood have been shown to be associated with the progression of CNV [13]. Previously, we have identified the reduction in IL-6 levels (more significantly than any other cytokine) in human aqueous samples after intravitreal injection of bevacizumab in CNV associated with exudative AMD [14]. However, concurrent correlation between aqueous levels of VEGF and IL-6 and its relation to clinical outcome after intravitreal bevacizumab are not known in patients with active CNV associated with exudative AMD.

In this study, we measured IL-6 and VEGF levels in aqueous samples of patients with CNV prior to and after administration of intravitreal bevacizumab to evaluate its efficacy and attempted to evaluate relationship between the cytokine levels and clinical outcome.

\section{Methods}

In this prospective, non-randomized interventional study, we measured aqueous cytokines including VEGF and IL-6 in patients undergoing intravitreal anti-VEGF treatment for exudative AMD. Approval for this study was obtained from Institutional Review Board and an informed consent was obtained from all the participating subjects. The control group included 30 eyes from 30 patients undergoing cataract surgery without any other concurrent eye disease and without any prior intraocular surgery. The study group consisted of 30 eyes from 30 patients diagnosed with exudative AMD clinically and confirmed with flourescein angiogram and spectral-domain optical coherence tomography (SD-OCT, Spectralis, Heidelberg Engineering., Heidelberg, Germany). The inclusion criteria for the study group consisted of active choroidal neovascular membrane, with a washout period of 3 months from any previous treatment, age greater than 60 years and ability to provide an informed consent. The exclusion criteria for both groups were presence of other concurrent retinal pathology and presence of disciform scars.

All the study subjects underwent intravitreal injection of bevacizumab (Avastin ${ }^{\circledR}$ : Genentech, San Francisco, CA), $1.25 \mathrm{mg}$ in $0.05 \mathrm{ml}$, repeated three times at four weeks interval (baseline, 4 and 8 weeks). An ophthalmic examination including recording of best-corrected Snellen visual acuity and measurement of intraocular pressure was obtained prior to first injection (baseline) and after the third injection ( 8 weeks). All the study patients un- derwent macular imaging with Spectralis OCT at the above mentioned time points. Central subfield macular thickness (CSF thickness) was obtained from the data analyzed by the machine.

\subsection{Samples}

For the study group, aqueous humor was obtained from 30 patients undergoing bevacizumab injections for exudative AMD. The first sample was acquired before the first intravitreal bevacizumab injection, representing the baseline sample. The second sample was obtained prior to the third intravitreal injection of bevacizumab, to evaluate the effect of the treatment with 2 doses of bevacizumab on IL-6 and VEGF levels. Aqueous humor (80 $100 \mu \mathrm{L}$ ) was obtained through a limbal paracentesis site using a 27-gauge needle on a tuberculin syringe. Aqueous samples were collected without touching intraocular tissues. The samples were immediately frozen to prevent protein denaturation and stored at $-80^{\circ} \mathrm{C}$. 30 control samples were similarly acquired from age-matched patients without any known retinal pathology, undergoing cataract surgery.

\subsection{Multiplex Analysis}

Multiplex analysis was preformed to discern biomarker patterns within small sample volumes. The aqueous samples were thawed at room temperature, vortexed, and then spun at $1400 \mathrm{rpm}$ for $10 \mathrm{~min}$ to remove any precipitates. Subsequently, samples were diluted 1:3 and processed for IL-6 and VEGF analysis using singleplex bead immunoassay (Invitrogen Corporation, US). Standards were reconstituted and serially diluted, per the manufacturer's instructions. A 96-well microtiter plate was used and $25 \mu \mathrm{L}$ of bead solution was added to each designated well and incubated for 30 seconds. After incubation, the supernatant was aspirated, using a vacuum manifold; 50 $\mu \mathrm{L}$ of incubation buffer was added to each well prior to $50 \mu \mathrm{L}$ of assay diluent and $50 \mu \mathrm{L}$ of the aqueous sample. Diluted standards were then added to the designated wells and the plate was then incubated for 2 hours at room temperature on an orbital shaker (250 - 500 rpm). After incubation, the liquid was removed and the excess beads were washed repeatedly. Furthermore, $100 \mu \mathrm{L}$ of detector antibody, specific for VEGF and IL-6, was added to each well. The plate was incubated on an orbital shaker at room temperature for an hour. After incubation, excess detector antibodies were removed and substrate complex (S-RPE: Streptavidin R-Phycoerythrin) was added. Luminex $100^{\mathrm{TM}}$ IS fluoroanalyzer (Luminex Inc., Austin, TX, US) was used to measure the fluorescence after 30 minutes of incubation at room temperature. To ensure proper assay, a known concentration of human 
recombinant VEGF as well as IL-6 was included in each run as a positive control.

The time points in the study were at baseline and at 8 weeks. The primary outcomes of this experiment were measurement of aqueous VEGF and IL-6 concentrations in the study group before and after treatment with 2 doses of intravitreal bevacizumab. The secondary outcomes consisted of correlation of change in aqueous VEGF and IL-6 concentrations with change in visual acuity and CSF thickness.

\subsection{Statistics}

Statistical analysis was performed with GraphPad Instat (GraphPad Software, Inc, CA, US). Snellen visual acuity was converted to LogMar for analysis. Baseline parameters were compared using the chi-square test; changes in aqueous VEGF and IL-6 values (at baseline and at 8 weeks) were analyzed using Wilcoxon rank sum test and correlation statistics were performed using Spearman correlation test.

\section{Results}

Baseline characteristics of the control and study patients are as shown in Table 1 . Thirty patients were included in the control group with a mean age of $72.8 \pm 7.0$ years. 30 patients diagnosed with exudative AMD formed the study group with a mean age of $73.7 \pm 8.6$ years. The study group had predominantly male patients (female/ male 7:23) and patients were predominantly caucasian (African American/Caucasian 1:29).

Study patients had significantly higher VEGF and IL-6 levels compared to controls. Mean aqueous VEGF concentration in the study group was $6 \pm 3$ picograms (pg) compared to $3.7 \pm 1.3 \mathrm{pg}$ in controls $(\mathrm{p}=0.001)$. Similarly, mean aqueous IL-6 concentration in the study group was $17.1 \pm 21.3 \mathrm{pg}$ compared to $5.1 \pm 2.2 \mathrm{pg}$ in controls $(\mathrm{p}<0.0001)$.

In the study, best-corrected visual acuity at baseline was $-0.52 \pm 0.65 \log M a r$ and at 8 weeks was $0.58 \pm 0.62$ $\operatorname{logMar}(\mathrm{p}=0.43)$. Mean baseline intraocular pressure was $14.3 \pm 3.4 \mathrm{mmHg}$ and following treatment was 13.9 $\pm 3.7 \mathrm{mmHg}(\mathrm{p}=0.5)$.

The mean baseline CSF thickness was $443.7 \pm 215.7$ microns and at 8 weeks was $370.5 \pm 143.6$ microns representing a change of $11.5 \% \pm 20.8 \%(p=0.009)$. Visual acuity correlated poorly with change in CSF thickness $(r=0.01)$.

There was no significant difference in aqueous VEGF levels $(p=0.38)$ between baseline and 8 weeks post injection, although levels decreased in most cases and a decreasing trend was discernible (Figure 1, left panel). Post treatment aqueous VEGF levels showed poor corre- lation with change in CSF thickness $(r=0.19, p=0.32)$. Similar to aqueous VEGF concentration, there was no significant difference between baseline and 8 weeks post injection in aqueous IL-6 levels $(p=0.12)$ (Figure 1, right panel). Similar to aqueous VEGF, there was a decreasing trend in aqueous IL-6 levels observed following treatment. However, change in aqueous IL-6 levels showed significant correlation with change in CSF thickness $(r=0.64, p=0.002)$.

Analyses of data revealed that there was no uniform resolution of macular thickening on Spectralis OCT following intravitreal bevacizumab treatment. The study patients were then sub-grouped based on their CSF thickness change. Group 1 included patients $(n=14)$ who had a decrease in CSF thickness greater than $10 \%$ from the baseline and were categorized to have "improved”. Group 2 included patients $(\mathrm{n}=16)$ with a decrease in CSF thickness $10 \%$ or less and were considered "treatment-resistant".

In the subanalysis, in Group 1 patients, mean change in visual acuity was $0.0 \pm 0.27 \log$ Mar. Mean CSF thickness at baseline was $506.7 \pm 238.5$ microns and at 8 weeks was $362.6 \pm 133.1$ microns $(\mathrm{p}=0.001)$. Aqueous VEGF concentrations at 8 weeks was $5.6 \pm 2.1 \mathrm{pg}$ compared to $5.7 \pm 2.1 \mathrm{pg}$ at baseline $(\mathrm{p}=0.97)$ (Figure 2, left panel). Similarly, aqueous IL-6 concentration at 8 weeks was $12.7 \pm 7.2 \mathrm{pg}$ compared to $23.3 \pm 25.9 \mathrm{pg}$ at baseline $(p=0.02)$ (Figure 2, right panel). There was a stronger correlation of change in CSF thickness with aqueous IL-6 levels $(r=0.72)$ compared with aqueous VEGF levels $(r=0.42)$ (Figure 3).

In Group 2 patients, mean change in visual acuity was $-0.14 \pm 0.61 \log$ Mar. Mean CSF thickness before treatment was $376.2 \pm 171.6$ microns and at 8 weeks was $378.9 \pm 158.5$ microns $(\mathrm{p}=0.1)$. Aqueous VEGF concentrations was $5.5 \pm 2.5 \mathrm{pg}$ compared to $6.5 \pm 3.7 \mathrm{pg}$ at baseline $(p=0.5)$ (Figure 4, left panel). Similarly, aqueous IL-6 concentration was $14.6 \pm 15.5$ pg compared to $10.4 \pm 12.8 \mathrm{pg}$ at baseline $(\mathrm{p}=0.006)$ (Figure 4, right panel). Similar to Group 1, IL-6 showed stronger correlation with change in CSF thickness compared to aqueous VEGF levels ( $r=0.53$ and 0.79 , respectively) (Figure 5).

\section{Discussion}

In this study, we investigated the correlation between VEGF and IL-6 levels in the aqueous with the treatment response after intravitreal bevacizumab for exudative AMD. Aqueous IL-6 levels showed a better correlation than aqueous VEGF levels in predicting treatment response in exudative AMD after bevacizumab treatment.

Exudative AMD, characterized by choroidal neovascularization is driven by ischemia induced upregulation of VEGF [4]. Increased levels of VEGF in the aqueous 
Table 1. Baseline characteristics of study and control population.

\begin{tabular}{ccc}
\hline & Control group & Study group \\
\hline Age (in years) & $72.8 \pm 7.0$ & $73.7 \pm 8.6$ \\
Sex (female/male) & $4: 26$ & $7: 23$ \\
Race (African American/ Caucasian) & $26: 4$ & 0.1 \\
Aqueous VEGF levels (in picogram) & $3.7 \pm 1.3$ & $6 \pm 3$ \\
Aqueous IL-6 levels (in picogram) & $5.1 \pm 2.2$ & 0.001 \\
\hline
\end{tabular}
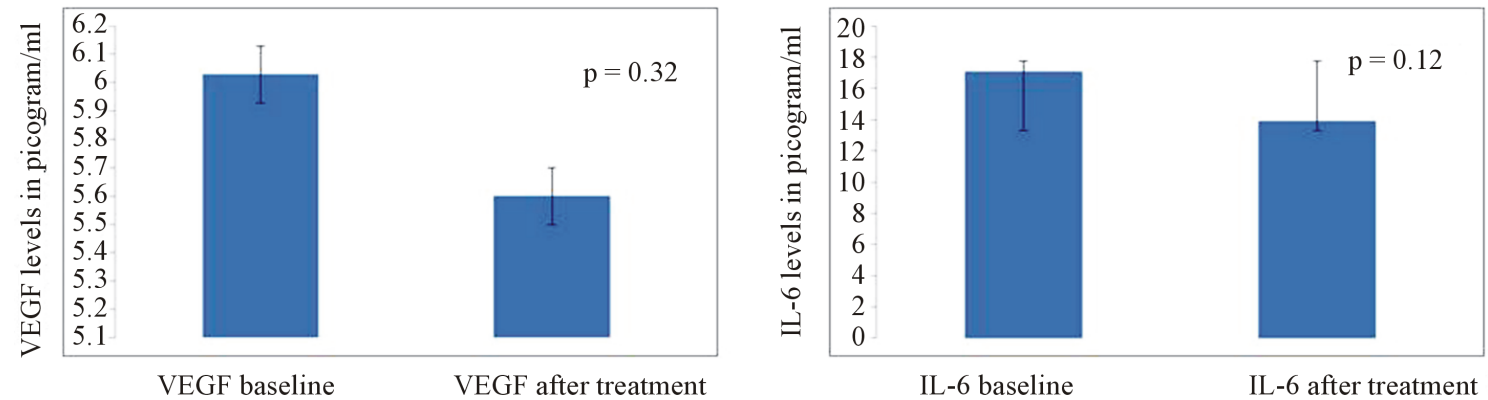

Figure 1. Column chart showing the change in mean aqueous VEGF levels (left) and change in mean aqueous IL-6 levels (right) in the study patients. The error bar represents standard deviation.
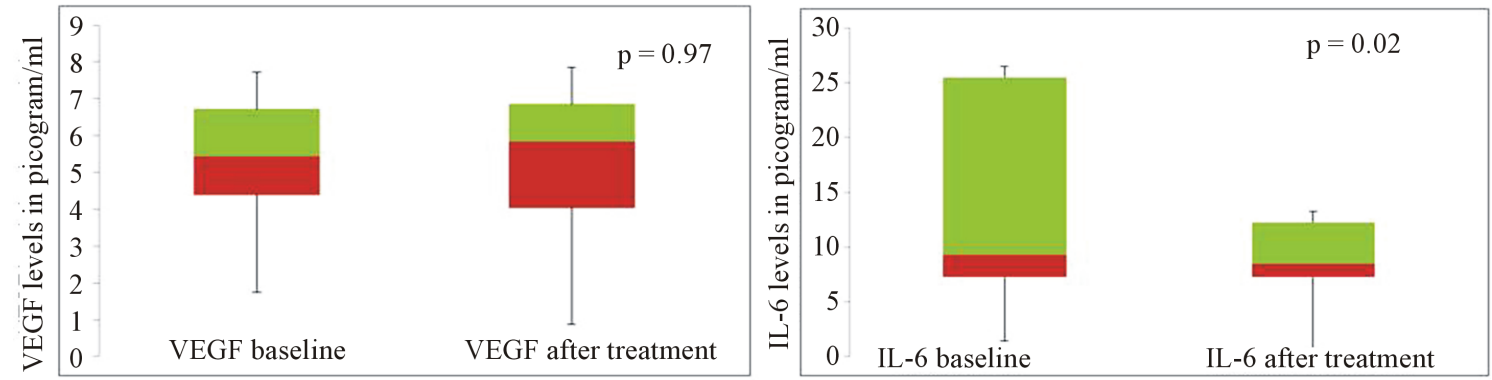

Figure 2. Box plot diagram showing the change in mean aqueous VEGF levels (left) and change in mean aqueous IL-6 levels (right) in patients in Group 1. The error bar represents standard deviation.

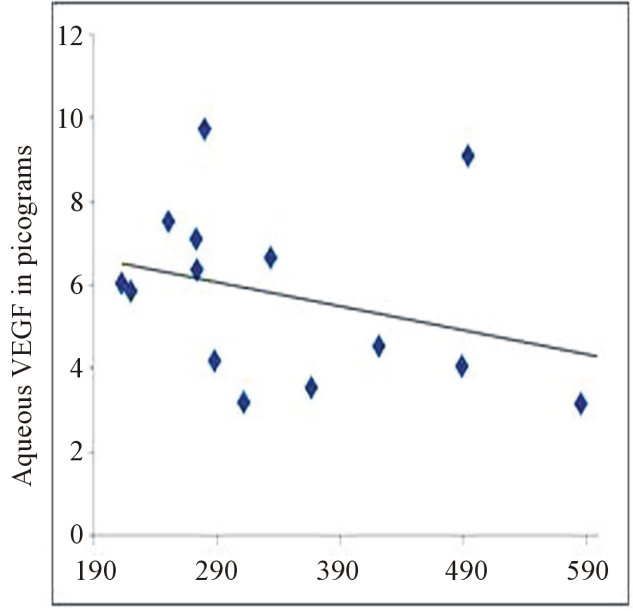

Central subfield thickness in microns

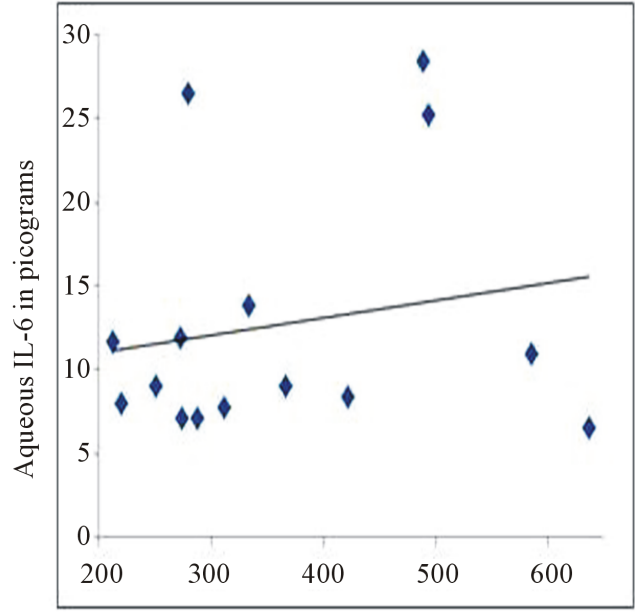

Central subfield thickness in microns

Figure 3. Correlation plot showing correlation between aqueous VEGF (left) and aqueous IL-6 levels (right) with central subfield thickness on Spectralis OCT in patients in Group 1. 

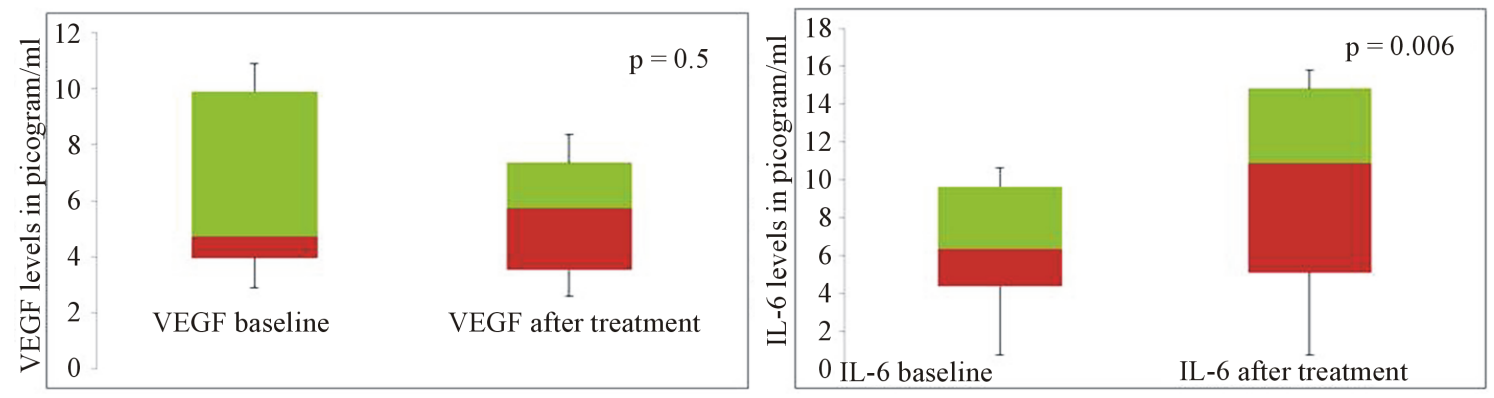

Figure 4. Box plot diagram showing the change in mean aqueous VEGF levels (left) and change in mean aqueous IL-6 levels (right) in patients in Group 2. The error bar represents standard deviation.

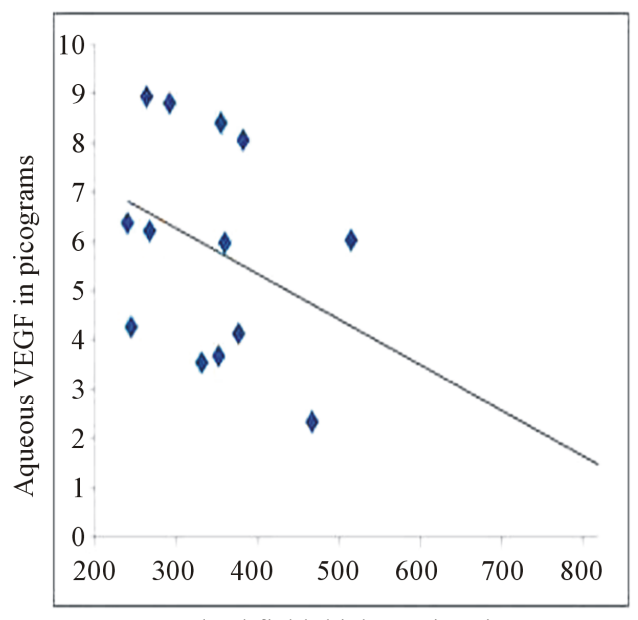

Central subfield thickness in microns

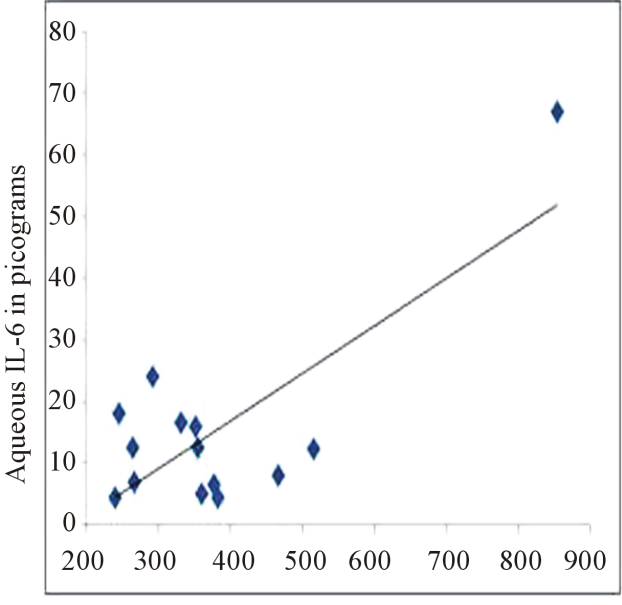

Central subfield thickness in microns

Figure 5. Correlation plot showing correlation between aqueous VEGF (left) and aqueous IL-6 levels (right) with central subfield thickness on Spectralis OCT in patients in Group 2.

are observed in several ocular ischemic conditions including diabetic retinopathy, retinal vascular occlusions and exudative AMD [5]. In our study, we measured cytokines level using bead-based immunoassay with flow cytometry, which we previously have shown to accurately measure VEGF concentration in micro-samples [15]. Besides ischemia, inflammation has been implicated in the etiopathogenesis of CNV [9]. IL-6, a key inflammatory mediator, is a multifunctional cytokine that can indirectly increase vascular permeability by inducing VEGF expression and directly increases endothelial permeability [11]. Similar to VEGF, aqueous IL-6 levels have been found to be increased in patients with ischemic retinal conditions [16]. In patients with active CNV, Roh et al. have reported significant correlation between the aqueous humor levels of IL-6 and the size of CNV [17]. However, in their study, IL-6 levels in the aqueous measured in patients with treatment-naïve $\mathrm{CNV}$, patients with recurrent $\mathrm{CNV}$ and patients with regressed $\mathrm{CNV}$ were not significantly different from that measured in the control group.

Aqueous VEGF levels have been shown to normalize after intravitreal bevacizumab in retinal conditions such as diabetic macular edema and retinal vascular occlusions [18]. However, similar correlation has not been established in exudative AMD. In our study, no statistical significant reduction in aqueous VEGF and IL-6 levels was found after intravitreal bevacizumab treatment, though both cytokine levels showed a decreasing trend. Roh et al. have reported that the concentration of aqueous cytokines (including IL-2, 4, 6, 8, 10, TNF- $\alpha$ ) were found to be unchanged after consecutive intravitreal bevacizumab [19]. Focality of disease process in AMD is one possible explanation for the poor correlation of aqueous VEGF levels with the treatment response.

In ischemic retinal conditions, aqueous VEGF levels have been shown to correlate with the severity of the disease process, which is measured clinically with visual acuity or the macular thickness on OCT [16]. In the study, treatment response was defined based on the change in central macular thickness noted on sequential scans done at 8 weeks apart on Spectralis OCT. Studies correlating visual acuity outcomes after anti-VEGF treatment with retinal parameters on SD-OCT have shown that central 
retinal thickness correlates poorly with visual acuity [20]. Retinal scans obtained on SD-OCT have been further delineated using segmentation algorithms to show that visual acuity correlates better with outer retinal layer thickness in the fovea after anti-VEGF treatment in patients with exudative AMD [21]. However, these segmentation algorithms are not uniformly applied in clinical practice. In our study we used central subfield macular thickness (CSF thickness), which is the average thickness measured within the inner circle of $1-\mathrm{mm}$ diameter, to follow response to treatment. Previously, studies reporting normative data on macular thickness have reported a high repeatability of CSF thickness with a coefficient of correlation less than $9 \%$, with values above it representing a true onset of macular thickening [22]. Extrapolating from the same guideline for exudative AMD, we categorized treatment response to intravitreal bevacizumab as a decrease in CSF thickness of more than $10 \%$ from baseline and a treatment resistant group as a decrease in CSF thickness of $10 \%$ or less. In our study, a statistically significant correlation was found between IL-6 levels and CSF thickness in both treatmentresponsive and treatment-resistant groups, with stronger correlation in patients who showed treatment-resistance with intravitreal bevacizumab. On the other hand, no significant correlation was found in aqueous VEGF and CSF thickness in either group. The exact mechanism for increase in aqueous IL-6 levels after intravitreal bevacizumab cannot be explained. However, we hypothesize that there was an upregulation of the pathway upstream (principally the inflammatory cytokines such as IL-6) as bevacizumab neutralized the VEGF present intraocularly.

The strengths of our study include larger patient population, uniformity of treatment protocol and use of sensitive assays for measuring cytokines.

\section{Conclusion}

Data from our study suggest that aqueous IL-6 may be an important marker of treatment response. Future therapeutic strategies may include targeted treatment against both VEGF and IL-6, in patients who do not respond to antiVEGF treatment alone.

\section{REFERENCES}

[1] N. Congdon, B. O’Colmain, C. C. Klaver, R. Klein, B. Munoz, D. S. Friedman, J. Kempen, H. R. Taylor and P. Mitchell, Eye Diseases Prevalence Research Group, "Causes and Prevalence of Visual Impairment among Adults in the United States," Archives of Ophthalmology, Vol. 122, No. 4, 2004, pp. 477-485. http://dx.doi.org/10.1001/archopht.122.4.477

[2] J. Seddon and C. Chen, "The Epidemiology of Age Related Macular Degeneration,” In: J. Heier, Ed., Contro- versies and Advancement in the Treatment of Retinal Disease, Lippincott Williams \& Wilkins, Philadelphia, 2004, pp. 17-39.

[3] W. Richard Green, "Histopathology of Age-Related Macular Degeneration,” Molecular Vision, Vol. 5, 1999, pp. 27-36.

[4] E. J. Duh, H. S. Yang, J. A. Haller, E. De Juan, M. S. Humayun, P. Gehlbach, M. Melia, D. Pieramici, J. B. Harlan, P. A. Campochiaro and D. J. Zack, "Vitreous Levels of Pigment Epithelium-Derived Factor and Vascular Endothelial Growth Factor: Implications for Ocular Angiogenesis," American Journal of Ophthalmology, Vol. 137, No. 4, 2004, pp. 668-674.

[5] L. P. Aiello, R. L. Avery, P. G. Arrigg, B. A. Keyt, H. D. Jampel, S. T. Shah, L. R. Pasquale, H. Thieme, M. A. Iwamoto, J. E. Park, H. V. Nguyen, L. M. Aiello, N. Ferrara and G. L. King, "Vascular Endothelial Growth Factor in Ocular Fluid of Patients with Diabetic Retinopathy and Other Retinal Disorders," The New England Journal of Medicine, Vol. 331, No. 22, 1994, pp. 1480-1487. http://dx.doi.org/10.1056/NEJM199412013312203

[6] E. S. Gragoudas, A. P. Adamis, E. T. Cunningham Jr., M. Feinsod and D. R. Guyer, "Pegaptanib for Neovascular Age-Related Macular Degeneration," The New England Journal of Medicine, Vol. 351, No 27, 2004, pp. 28052816. http://dx.doi.org/10.1056/NEJMoa042760

[7] D. M. Brown, P. K. Kaiser, M. Michels, G. Soubrane, J. S. Heier, R. Y. Kim, J. P. Sy and S. Schneider, ANCHOR Study Group, "Ranibizumab versus Verteporfin for Neovascular Age-Related Macular Degeneration," The New England Journal of Medicine, Vol. 355, No. 14, 2006, pp. 1432-1444. http://dx.doi.org/10.1056/NEJMoa062655

[8] R. L. Avery, D. J. Pieramici, M. D. Rabena, A. A. Castellarin, M. A. Nasir and M. J. Giust, "Intravitreal Bevacizumab (Avastin) for Neovascular Age-Related Macular Degeneration,” Ophthalmology, Vol. 113, No. 3, 2006, pp. 363.e5-372.e5. http://dx.doi.org/10.1016/j.ophtha.2005.11.019

[9] A. Kijlstra, E. C. La Heij and F. Hendrikse, "Immunological Factors in the Pathogenesis and Treatment of AgeRelated Macular Degeneration,” Ocular Immunology and Inflammation, Vol. 13, No. 1, 2005, pp. 3-11. http://dx.doi.org/10.1080/09273940590909185

[10] A. Kvanta, P. V. Algvere, L. Berglin and S. Seregard, "Subfoveal Fibrovascular Membranes in Age-Related Macular Degeneration Express Vascular Endothelial Growth Factor," Investigative Ophthalmology \& Visual Science, Vol. 37, No. 9, 1996, pp. 1929-1934.

[11] T. Cohen, D. Nahari, L. W. Cerem, G. Neufeld and B. Z. Levi, "Interleukin 6 Induces the Expression of Vascular Endothelial Growth Factor," The Journal of Biological Chemistry, Vol. 271, No. 2, 1996, pp. 736-741. http://dx.doi.org/10.1074/jbc.271.2.736

[12] A. Saidi, M. Hagedorn, N. Allain, C. Verpelli, C. Sala, L. Bello, A. Bikfalvi and S. Javerzat, "Combined Targeting of Interleukin-6 and Vascular Endothelial Growth Factor Potently Inhibits Glioma Growth and Invasiveness,” International Journal of Cancer, Vol. 125, No. 5, 2009, pp. 
1054-1064. http://dx.doi.org/10.1002/ijc.24380

[13] J. M. Seddon, S. George, B. Rosner and N. Rifai, "Progression of Age-Related Macular Degeneration: Prospective Assessment of C-Reactive Protein, Interleukin 6, and Other Cardiovascular Biomarkers," Archives of Ophthalmology, Vol. 123, No. 6, 2005, pp. 774-782. http://dx.doi.org/10.1001/archopht.123.6.774

[14] R. K. Sharma, A. T. Rogojina and K. V. Chalam, "Bevacizumab Therapy Normalizes the Pathological Intraocular Environment beyond Neutralizing VEGF," Molecular Vision, Vol. 16, 2010, pp. 2175-2184.

[15] K. V. Chalam, S. Balaiya and R. K. Murthy, "Accurate Estimation of Vascular Endothelial Growth Factor Levels in Microsamples with a Low-Cost Bead-Based Assay,” Retina, Vol. 30, No. 5, 2010, pp. 815-819. http://dx.doi.org/10.1097/IAE.0b013e3181c70153

[16] H. Noma, H. Funatsu, M. Yamasaki, H. Tsukamoto, T. Mimura, T. Sone, T. Hirayama, H. Tamura, H. Yamashita, A. Minamoto and H. K. Mishima, "Aqueous Humour Levels of Cytokines Are Correlated to Vitreous Levels and Severity of Macular Oedema in Branch Retinal Vein Occlusion,” Eye, Vol. 22, No. 1, 2008, pp. 42-48. http://dx.doi.org/10.1038/sj.eye.6702498

[17] M. I. Roh, S. J. Lim, J. M. Ahn, J. B. Lim and O. W. Kwon, "Concentration of Cytokines in Age-Related Macular Degeneration after Consecutive Intravitreal Bevacizumab Injection," Graefe's Archive for Clinical and Experimental Ophthalmology, Vol. 248, No. 5, 2010, pp. 635-640. http://dx.doi.org/10.1007/s00417-009-1254-8

[18] M. Funk, G. Schmidinger, N. Maar, M. Bolz, T. Benesch and G. J. Zlabinger, U. M. Schmidt-Erfurth, “Angiogenic and Inflammatory Markers in the Intraocular Fluid of Eyes with Diabetic Macular Edema and Influence of
Therapy with Bevacizumab,” Retina, Vol. 30, No. 9, 2010, pp. 1412-1419. http://dx.doi.org/10.1097/IAE.0b013e3181e095c0

[19] M. I. Roh, H. S. Kim, J. H. Song, J. B. Lim, H. J. Koh and O. W. Kwon, "Concentration of Cytokines in the Aqueous Humor of Patients with Naive, Recurrent and Regressed CNV Associated with Amd after Bevacizumab Treatment," Retina, Vol. 29, No. 4, 2009, pp. 523529. http://dx.doi.org/10.1097/IAE.0b013e318195cb15

[20] P. A. Keane, S. Liakopoulos, K. T. Chang, M. Wang, L. Dustin, A. C. Walsh and S. R. Sadda, "Relationship between Optical Coherence Tomography Retinal Parameters and Visual Acuity in Neovascular Age-Related Macular Degeneration,” Ophthalmology, Vol. 115, No. 12, 2008, pp. 2206-2214. http://dx.doi.org/10.1016/j.ophtha.2008.08.016

[21] I. Golbaz, C. Ahlers, G. Stock, C. Schütze, S. Schriefl, F. Schlanitz, C. Simader, C. Prünte and U. M. SchmidtErfurth, "Quantification of the Therapeutic Response of Intraretinal, Subretinal, and Subpigment Epithelial Compartments in Exudative AMD during Anti-VEGF Therapy,” Investigative Ophthalmology \& Visual Science, Vol. 52, No. 3, 2011, pp. 1599-1605. http://dx.doi.org/10.1167/iovs.09-5018

[22] D. J. Browning, C. M. Fraser and B. W. Propst, "The Variation in Optical Coherence Tomography-Measured Macular Thickness in Diabetic Eyes without Clinical Macular Edema," American Journal of Ophthalmology, Vol. 145, No. 5, 2008, pp. 889-893. http://dx.doi.org/10.1016/j.ajo.2008.01.007 